\title{
The use of GPS arrays in detecting the ionospheric response during rocket launchings
}

\author{
Edward L. Afraimovich, Eugene A. Kosogorov, Kirill S. Palamarchouk, Natalia P. Perevalova, and Alexey V. Plotnikov \\ Institute of Solar-Terrestrial Physics SD RAS, P.O. Box 4026, Irkutsk 664033, Russia
}

(Received January 17, 2000; Revised August 10, 2000; Accepted September 19, 2000)

\begin{abstract}
In this paper we investigate the form and dynamics of shock acoustic waves (SAW) generated during the rocket PROTON launching from the Baikonur cosmodrome in 1998 and 1999. In spite of the difference of geophysical conditions, the ionospheric response for all launchings has a period of about $300 \mathrm{~s}$ and the amplitude exceeding background fluctuations under quiet and moderate geomagnetic conditions by factors of 2 to 5 as a minimum. The angle of elevation of the SAW wave vector varies from $45^{\circ}$ to $60^{\circ}$, and the SAW phase velocity $(900-1200 \mathrm{~m} / \mathrm{s})$ approaches the sound velocity at heights of the ionospheric F region maximum. The position of the SAW source, inferred by neglecting refraction corrections, corresponds to the segment of the rockets path at a distance no less than 700-900 km from the launch pad, which is consistent with the estimated delay time of SAW source triggering (250-300 s).
\end{abstract}

\section{Introduction}

A large number of publications (Arendt, 1971; Karlov et al., 1980; Mendillo, 1981, 1982; Noble, 1990, etc.) are devoted to the study of the ionospheric response of the shock wave produced during launchings of powerful rockets. Scientific interest in this problem is due to the fact that such launchings can be regarded as active experiments in the Earth's atmosphere, and they can be used in solving a wide variety of problems in the physics of the ionosphere and radio wave propagation. These investigations have also important practical implications since they furnish a means of substantiating reliable signal indications of technogenic effects (among them, unauthorized), which is necessary for the construction of an effective global radiophysical subsystem for detection and localization of these effects.

Published data on fundamental parameters of the shock acoustic waves (SAW) differ greatly. According to a review by Karlov et al. (1980), the oscillation period of the ionospheric response of the SAW, recorded during launchings of the APOLLO mission rockets, varied from 6 to $90 \mathrm{~min}$, and the propagation velocity was in the range from 600 to $1670 \mathrm{~m} / \mathrm{s}$. There is an even greater uncertainty in the localization of the region of SAW generation, which is necessary both for an understanding of the physical mechanisms of the phenomenon, and for a scientific justification of the solution of applied problems. Published data are discussed in more detail in Section 5, by comparison with our results.

The lack of comprehensive, reliable data on SAW parameters is due primarily to the limitations of existing experimental methods and detection facilities. The main body of data was obtained by measuring the frequency Doppler shift at

Copy right $(\mathcal{C}$ The Society of Geomagnetism and Earth, Planetary and Space Sciences (SGEPSS); The Seismological Society of Japan; The Volcanological Society of Japan; The Geodetic Society of Japan; The Japanese Society for Planetary Sciences. vertical and oblique-incidence ionospheric soundings in the HF range (Jacobson and Carlos, 1994). In some instances the sensitivity of this method is sufficient to detect the SAW reliably; however, difficulties emerge when localizing the region where the detected signal is generated. These problems are caused by the multiple-hop character of HF signal propagation.

Currently some authors have embarked on an intense development of methods for the use of GPS data in detecting the ionospheric response of strong earthquakes (Calais and Minster, 1995), rocket launchings (Calais and Minster, 1996), and industrial surface explosions (Fitzgerald, 1997; Calais et al., 1998). In the cited references the SAW phase velocity was determined by the 'crossing' method by estimating the time delay of SAW arrival at subionospheric points corresponding to different GPS satellites observed at a given time. However, the accuracy of such a method is rather low because the altitude at which the subionospheric points are specified, is determined in a crude way.

The goal of this paper is to develop a method for determining SAW parameters (including the phase velocity, angular characteristics of the SAW wave vector, the direction towards the source, and the source location) using GPS arrays whose elements can be chosen out of a large set of GPS stations of a global GPS network (Klobuchar, 1997). Contrary to existing radio techniques, this method estimates SAW parameters without a priori information about the location and time of a rocket launching.

\section{The Geometry and General Characterization of Experiments}

This paper presents the results derived from determining the key SAW parameters during ascents of the PROTON rockets from cosmodromes Baikonur $\left(45.6^{\circ} \mathrm{N}, 63.3^{\circ} \mathrm{E}\right)$ in 1998 and 1999 (a total of three launchings). Figure 1 por- 
Table 1. Parameters of rocket launch-induced SAWs.

\begin{tabular}{|c|c|c|c|c|c|c|c|c|c|c|c|c|c|}
\hline Sites & $\begin{array}{c}t_{p} \\
(\mathrm{UT})\end{array}$ & $\begin{array}{c}\Delta t \\
(\mathrm{sec} .)\end{array}$ & $\begin{array}{c}T \\
\text { (sec.) }\end{array}$ & $\begin{array}{c}A_{I} \\
(\mathrm{TECU})\end{array}$ & $\begin{array}{c}A_{F} \\
(\mathrm{~Hz})\end{array}$ & $\begin{array}{l}\alpha \\
\left(^{\circ}\right)\end{array}$ & $\begin{array}{c}\theta \\
\left(^{\circ}\right)\end{array}$ & $\begin{array}{c}V_{h} \\
(\mathrm{~m} / \mathrm{s})\end{array}$ & $\begin{array}{c}V_{t} \\
(\mathrm{~m} / \mathrm{s})\end{array}$ & $\begin{array}{c}V_{\alpha} \\
(\mathrm{m} / \mathrm{s})\end{array}$ & $\begin{array}{l}\phi_{w} \\
\left(^{\circ}\right)\end{array}$ & $\begin{array}{l}\lambda_{w} \\
\left({ }^{\circ}\right)\end{array}$ & $\begin{array}{c}\Delta t_{w} \\
\text { (sec.) }\end{array}$ \\
\hline \multicolumn{14}{|c|}{ July 5,$1999 ; t_{0}=13: 32$ UT; PRN 14; DAY 186; Dst $=11 \mathrm{nT}$} \\
\hline SELE & $13: 44: 44$ & 764 & 300 & 0.46 & 0.12 & 58.9 & 152.8 & 1808 & 931 & 1070 & 48 & 66 & 264 \\
\hline \multicolumn{14}{|l|}{ CHUM } \\
\hline \multicolumn{14}{|l|}{ SHAS } \\
\hline SUMK & $13: 42: 44$ & 644 & 300 & 0.75 & 0.17 & 58 & 157 & 1639 & 874 & 976 & 47.9 & 66 & 259 \\
\hline \multicolumn{14}{|l|}{ POL2 } \\
\hline \multicolumn{14}{|l|}{ SELE } \\
\hline KUMT & $13: 45: 54$ & 834 & 360 & 0.36 & 0.08 & 59 & 152 & 2473 & 1271 & 1094 & 47.7 & 66.7 & 265 \\
\hline \multicolumn{14}{|l|}{ SHAS } \\
\hline \multicolumn{14}{|l|}{ SELE } \\
\hline POL2 & $13: 44: 32$ & 752 & 300 & 0.39 & 0.11 & 58.7 & 154 & 2458 & 1278 & 894 & 47.8 & 66 & 262 \\
\hline \multicolumn{14}{|l|}{ SELE } \\
\hline \multicolumn{14}{|l|}{ KUMT } \\
\hline CHUM & $13: 44: 09$ & 729 & 300 & 0.49 & 0.13 & 58.8 & 153.6 & 1741 & 901 & 926 & 48 & 66 & 263 \\
\hline \multicolumn{14}{|l|}{ SELE } \\
\hline \multicolumn{14}{|l|}{ SUMK } \\
\hline$\sum$ & & & 305 & 0.48 & 0.12 & 58.7 & 153.9 & 2024 & 1051 & 992 & 47.9 & 66.1 & 263 \\
\hline \multicolumn{14}{|c|}{ October 27, 1999; $t_{0}=16: 16$ UT; PRN 9; DAY 300; Dst $=-80 \mathrm{nT}$} \\
\hline \multicolumn{13}{|l|}{$\begin{array}{l}\text { POL2 } \\
\text { SHAS }\end{array}$} & 308 \\
\hline SHAS & & & & & & & & & & & & & \\
\hline SUMK & $16: 29: 31$ & 811 & 270 & 0.12 & 0.038 & 46.3 & 162 & 1666 & 1151 & 827 & 47.4 & 68.4 & 325 \\
\hline \multicolumn{14}{|l|}{ POL2 } \\
\hline \multicolumn{14}{|l|}{ SHAS } \\
\hline SHAS & $16: 31: 31$ & 931 & 300 & 0.053 & 0.016 & 45.9 & 163 & 1659 & 1155 & 844 & 47.5 & 68.4 & 302 \\
\hline \multicolumn{14}{|l|}{ CHUM } \\
\hline \multicolumn{14}{|l|}{ SUMK } \\
\hline POL2 & $16: 31: 18$ & 918 & 270 & 0.067 & 0.017 & 46.8 & 161 & 1666 & 1151 & 811 & 47.4 & 67.7 & 312 \\
\hline \multicolumn{14}{|l|}{ CHUM } \\
\hline \multicolumn{14}{|l|}{ SUMK } \\
\hline$\sum$ & & & 285 & 0.08 & 0.02 & 46.4 & 162 & 1668 & 1153 & 826 & 47.5 & 68 & 312 \\
\hline \multicolumn{14}{|c|}{ November 20, 1998; $t_{0}=06: 40$ UT; PRN 16; DAY 324; Dst $=-9$ nT } \\
\hline CHUM & $6: 53: 42$ & 822 & 300 & 0.11 & 0.028 & 52.8 & 162.9 & 1338 & 809 & 890 & 48.3 & 67.7 & 308 \\
\hline SELE & $6: 54: 06$ & 846 & 300 & 0.09 & 0.02 & & & & & & & & \\
\hline POL2 & $6: 54: 06$ & 846 & 300 & 0.08 & 0.017 & & & & & & & & \\
\hline
\end{tabular}




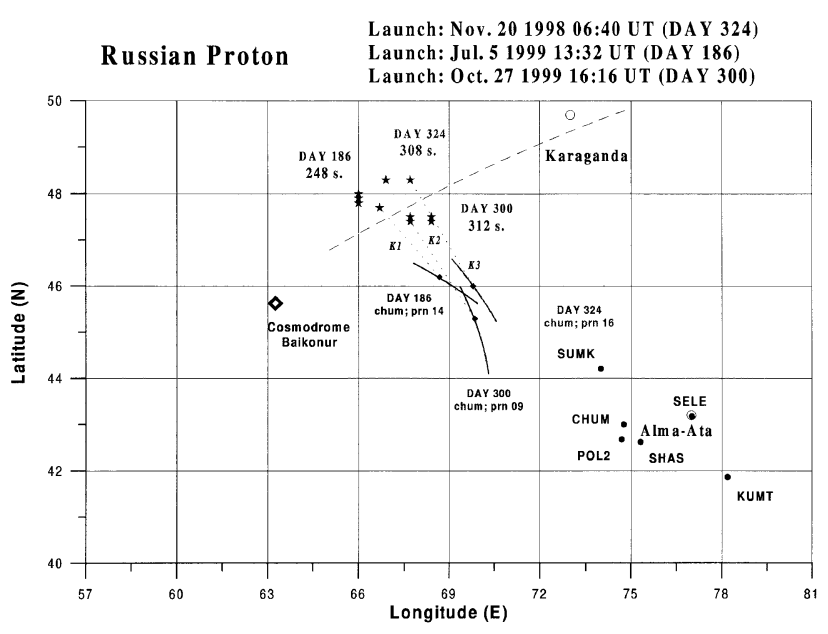

Fig. 1. Experimental geometry during PROTON launches from Baikonur Cosmodrome. Solid lines show trajectories of subionospheric points for each GPS satellite at the height $h_{\max }=400 \mathrm{~km}$. Dark diamonds along the trajectories show the position of subionospheric points at the time $t_{\mathrm{p}}$ of a maximum deviation of the frequency Doppler shift $F$. Asterisks show the source position at $100 \mathrm{~km}$ altitude determined using the data from the GPS-arrays. Numbers at the asterisks correspond to day numbers and to the 'switch-on' delay of the source with respect to the start time. Dashed straight lines connecting the anticipated source with the subionospheric point designate the horizontal projection of the wave vector $\boldsymbol{K}$. Heavy dots and upper-case letters mark the position and names of the GPS stations, while lower-case letters along the trajectories refer to station names and PRN numbers of the GPS satellites.

trays the geometry of experiments. The dashed line roughly corresponds to the horizontal projection of the rocket flight trajectory with orbital inclination $\psi=51.6^{\circ}$. The symbol $\diamond$ marks the pad site.

Below is a summary of results derived from detecting one standard launching (on November 20, 1998) and two abortive launchings (July 5 and October 27, 1999) of the PROTON. Information about rocket launchings from the Baikonur Cosmodrome can be extracted from the Internet sites: http//www.flatoday. com; http: //www.ksc. nasa.gov; http://www.spacelaunchnews.com; and http: //www. isllaunch. com.

General information regarding these launches are summarized in Table 1 (including the start time $t_{0}$ of UT and the level of geomagnetic disturbance according to geomagnetic index Dst). It was found that the deviation of Dst for the selected days was quite moderate thus enabling the SAW to be identified.

\section{Methods of Determining Shock Acoustic Wave Characteristics Using GPS Arrays}

The standard GPS technology provides a means for wave disturbances detecion based on phase measurements of total electron content (TEC) at each of spaced two-frequency GPS receivers. A method of reconstructing TEC variations $I$ from measurements of the ionosphere-induced additional increment of the group and phase delay of the satellite radio signal was detailed and validated in a series of publications (Calais and Minster, 1995, 1996; Fitzgerald, 1997; Afraimovich et al., 1998).

GPS phase measurements can be made with a high degree of accuracy corresponding to the error of TEC determination of at least $10^{14} \mathrm{~m}^{-2}$ when averaged on a 30 -second time interval, with some uncertainty of the initial value of TEC, however (Hofmann-Wellenhof et al., 1992). This makes the detection of ionization irregularities and wave processes in the ionosphere possible over a wide range of amplitudes (up to $10^{-4}$ of the diurnal TEC variation) and periods (up to 1$5 \mathrm{~min}$ ). The unit of TEC (TECU), which is equal to $10^{16}$ $\mathrm{m}^{-2}$ and is commonly accepted in the literature, will be used in the following.

In some instances a convenient way of detecting and determining the ionospheric response delay of the shock wave involves inferring the frequency Doppler shift $F$ from TEC series $I$. Relevant results derived from analyzing the $F(t)$ variations calculated for the 'reduced' frequency of $136 \mathrm{MHz}$ are discussed in Section 4.

The correspondence of space-time phase characteristics, obtained through transionospheric soundings, with local characteristics of disturbances in the ionosphere was considered in detail in a wide variety of publications (Bertel et al., 1976; Afraimovich et al., 1992; Mercier and Jacobson, 1997) and is not analyzed at length in this study. The most important conclusion of the cited references is the fact that, as for the extensively exploited model of a 'plane phase screen' disturbances $\Delta I(x, y, t)$ of TEC faithfully copy the horizontal part of the corresponding disturbance of local concentration, independently of the angular position of the source, and can be used in experiments on measuring the wave disturbances of TEC.

However, the TEC response amplitude experiences a strong azimuthal dependence caused by the integral character of a transionospheric sounding. As a first approximation, the transionospheric sounding method is responsive only to the wave disturbances with the wave vector $\boldsymbol{K}_{t}$ perpendicular to the direction $\boldsymbol{r}$. A corresponding condition for elevation $\theta$ and azimuth $\alpha$ of an arbitrary wave vector $\boldsymbol{K}_{\boldsymbol{t}}$ normal to the direction $\boldsymbol{r}$, has the form

$$
\theta=\arctan \left(-\cos \left(\alpha_{s}-\alpha\right) / \tan \theta_{s}\right)
$$

where $\alpha_{s}$ - the azimuthal angle, counted off from the northward in a clockwise direction; and $\theta_{s}$ - the angle of elevation between the direction $\boldsymbol{r}$ and the terrestrial surface at the reception site.

We used formula (1) in determining the elevation $\theta$ of $\boldsymbol{K}_{\boldsymbol{t}}$ from the known mean value of azimuth $\alpha$ (see below). $\alpha$ and $\theta$ are reckoned from the northward direction and from the ground, respectively.

We now summarize briefly the sequence of data processing procedures. Out of a large number of GPS stations, three sites (A, B, C) are selected, the distances between which do not exceed about one-half the expected wavelength of the perturbation. Site B is taken to be the center of a topocentric reference frame whose axis $x$ is directed eastward, and the axis $y$ is directed northward. Such a configuration of the GPS receivers represents the GPS array with a minimum number of the required elements. In regions with a dense network of GPS sites, we can obtain a large variety of GPS arrays of a different configuration enabling the acquired data to be checked for reliability; in this paper we have exploited such a possibility. 
The input data include series of the 'oblique' value of TEC $I_{\mathrm{A}}(t), I_{\mathrm{B}}(t), I(t)$, as well as corresponding series of values of the elevation $\theta_{\mathrm{s}}(t)$ and the azimuth $\alpha_{\mathrm{s}}(t)$ of the beam to the satellite calculated, using our developed CONVTEC software which converts the RINEX files, standard for the GPS, from the INTERNET. For determining SAW characteristics, continuous series of measurements of $I_{\mathrm{A}}(t), I_{\mathrm{B}}(t), I_{\mathrm{C}}(t)$ are selected with a length of at least a one-hour interval which includes the start time.

To eliminate variations of the regular ionosphere, as well as trends introduced by orbital motion of the satellite, a procedure is used to remove the linear trend involving a preliminary smoothing of the initial series with the selected time window.

Series of the values of the elevation $\theta_{\mathrm{S}}(t)$ and azimuth $\alpha_{\mathrm{s}}(t)$ of the beam to the satellite are used to determine the location of the subionospheric point, as well as to calculate the elevation $\theta$ of the wave vector $\boldsymbol{K}_{t}$ disturbance from the known azimuth $\alpha$ (see formula (1)).

Figure 2 shows typical time dependencies of the TEC at the GPS array CHUM station near the Baikonur Cosmodrome.

It is evident from Fig. 2 that fast oscillations with a typical period $T$ of about $300 \mathrm{~s}$. are clearly distinguished among slow TEC variations. The oscillation amplitude $A$ (up to $0.5 \mathrm{TECU}$ ) is far in excess of the TEC fluctuation intensity during 'background' days. Variations in frequency Doppler shift $F(t)$ for spatially separated sites (SELE, CHUM, SHAS) are well correlated but are shifted relative to each other by an amount well below the period, which permits the SAW propagation velocity to be unambiguously determined.

With proper account of a good signal/noise ratio (larger than 1), we determine the horizontal projection of the phase velocity $V_{h}$ with the known coordinates of array sites A, B, C from time $t_{p}$ shifts of a maximum deviation of the TEC. Preliminarily measured shifts are subjected to a linear transformation with the purpose of calculating shifts for sites spaced relative to the central site northward $N$ and eastward $E$. This is followed by a calculation of the $E$ - and $N$-components of $V_{x}$ and $V_{y}$, as well as the direction $\alpha$ in the range of angles $0^{\circ}-360^{\circ}$ and the modulus $V_{h}$ of the horizontal component of the SAW phase velocity.

From the delay $\Delta t=t_{p}-t_{0}$ and the known path length between the launch pad and the subionospheric point we calculated also the SAW mean velocity $V_{a}$ in order to compare our obtained estimates of the SAW phase velocity with the usually used method of measuring this quantity.

The aspect condition (1) restricts the number of beam trajectories to the satellite, for which it is possible to detect reliably the SAW response in the presence of noise. On the other hand, formula (1) can be used to determine the elevation $\theta$ of the wave vector $\boldsymbol{K}_{\boldsymbol{t}}$ of the shock wave at the known value of the azimuth $\alpha$ (Afraimovich et al., 1998, 1999). Hence the phase velocity modulus $V_{t}$ can be defined as

$$
V_{t}=V_{h} \sec (\theta) .
$$

The ionospheric region that is responsible for the main contribution to TEC variations lies in the neighborhood of the maximum of the ionospheric $\mathrm{F}$ region, which does determine the height $h_{\max }$ of the subionospheric point. To a first approximation, it can be assumed that it is at this alti-

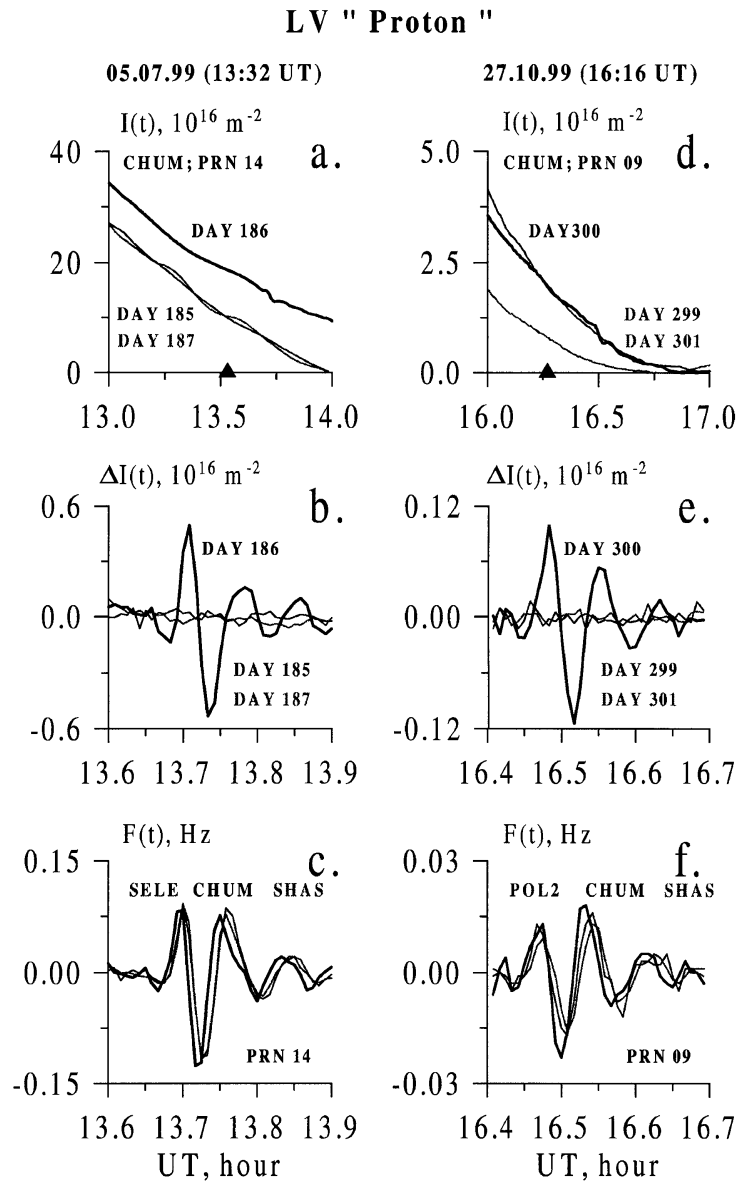

Fig. 2. Time dependencies of 'oblique' TEC $I(t)$ at one of the three sites of the GPS-array in the area of Baikonur Cosmodrome on the days of PROTOn launch (heavy curve), and one day before and one day after the start (thin curves) on July 5, 1999 — a, and on October 27, 1999—d; $\mathrm{b}$, e-for the same days but the TEC variations $\Delta I(t)$ with the linear trend removed and with a smoothing with a time window of $5 \mathrm{~min}$; c, $\mathrm{f}$ - variations of the frequency Doppler shift $F(t)$ 'reduced' to the sounding signal frequency of $136 \mathrm{MHz}$, for three sites of the arrays, start days. All panels show day numbers, GPS station names, and PRN numbers of the GPS satellites. The small arrows at the abscissa axis indicate the start time $t_{0}$.

tude where the imaginary detector is located, which records the ionospheric SAW response in TEC variations. When selecting $h_{\max }$ it should be taken into consideration that the decrease in electron density with height above the main maximum of the F2 layer proceeds much more slowly than is the case below the maximum. Since the density distribution with height is essentially a 'weight function' of the TEC response to a wave disturbance (Afraimovich et al., 1992), it is appropriate to use, as $h_{\max }$ the value exceeding the true height of the layer $h_{\mathrm{F} 2}$ maximum by about $100 \mathrm{~km}$. The $h_{\mathrm{F} 2}$ varies over a reasonably wide range (250-350 km) depending on the time of day and on some geophysical factors which, when necessary, can be taken into account if corresponding additional experimental data and current ionospheric models are used. In all calculations that follow, $h_{\max }=400 \mathrm{~km}$ is used.

From the GPS data we can determine the coordinates $X_{\mathrm{s}}$ and $Y_{\mathrm{S}}$ of the subionospheric point in the horizontal plane $X O Y$ of a topocentric frame of reference centered on the point $\mathrm{B}(0,0)$ at the time of a maximum TEC deviation caused 
by the arrival of the SAW at this point. Since we know the angular coordinates $\theta$ and $\alpha$ of the wave vector $\boldsymbol{K}_{\boldsymbol{t}}$, it is possible to determine the location of the point at which this vector intersects the horizontal plane $X^{\prime} O Y^{\prime}$ at the height $h_{\mathrm{w}}$ of the assumed source. Assuming a rectilinear propagation of the SAW from the source to the subionospheric point and neglecting the sphericity the coordinate $X_{\mathrm{w}} Y_{\mathrm{w}}$, and $h_{\text {max }}$ of the source in a topocentric frame of reference can be defined as

$$
\begin{aligned}
& X_{\mathrm{w}}=X_{\mathrm{p}}-\left(h_{\text {max }}-h_{\mathrm{w}}\right) \frac{\cos \theta \sin \alpha}{\sin \theta} \\
& Y_{\mathrm{w}}=Y_{\mathrm{p}}-\left(h_{\text {max }}-h_{\mathrm{w}}\right) \frac{\cos \theta \cos \alpha}{\sin \theta} .
\end{aligned}
$$

The coordinates $X_{\mathrm{w}}$ and $Y_{\mathrm{w}}$, thus obtained, can readily be recalculated to the values of the latitude and longitude $\left(\phi_{\mathrm{w}}\right.$ and $\lambda_{\mathrm{w}}$ ) of the source. When recording SAW produced by launchings of powerful rockets, the region of SAW generation can lie at heights $h_{\mathrm{w}}$ of order $100 \mathrm{~km}$ or higher (Li et al., 1994; Nagorsky, 1999).

Given the coordinates of the subionospheric point and of the disturbance source, the mean value of the SAW propagation velocity between the source and the subionospheric point, and the arrival time of the SAW at this point, then within the approximation of a rectilinear propagation it is easy to determine the 'switch-on' delay $\Delta t_{\mathrm{W}}$ of the anticipated SAW source with respect to the start. This would make it possible to obtain additional information about the SAW source which is needed to understand the mechanism of SAW generation. The estimates of $\Delta t_{\mathrm{w}}$ made below assume that the propagation velocity is taken equal to $700 \mathrm{~m} / \mathrm{s}$ (see Li et al., 1994). Note that by the 'switch-on' time of the source is meant here the time of a maximum disturbance of the background state of the medium when the SAW is generated.

Corresponding values of the SAW parameters, and also site names of the GPS array and GPS satellite PRN numbers are presented in Table 1.

\section{Results of Measurements}

Solid curves in Fig. 1 show trajectories of subionospheric points for each of the GPS satellites at the height $h_{\max }=$ $400 \mathrm{~km}$. Dark diamonds along the trajectories correspond to the coordinates of subionospheric points at the time $t_{\mathrm{p}}$ of a maximum deviation of the TEC. Asterisks designate the source location at $100 \mathrm{~km}$ altitude inferred from the GPS array data. Numbers at the asterisks refer to the corresponding day numbers and to the 'switch-on' delay of the source with respect to the start time. Dashed straight lines connecting the anticipated source with the subionospheric point show the horizontal projection of the wave vector $\boldsymbol{K}_{\boldsymbol{t}}$.

Let us consider the results derived from analyzing the ionospheric effect of SAW during failed PROTON launch on July 5, 1999 obtained at the array (SELE, CHUM,SHAS) for PRN14 (at the left of Fig. 2, and in Table 1).

In this case the delay of the SAW response with respect to the start time is $12 \mathrm{~min}$. The SAW has a period $T$ of about $300 \mathrm{~s}$. and an amplitude $A_{I}=0.5 \mathrm{TECU}$, which is an order of magnitude larger than TEC fluctuations for background days. It should be noted, however, that this time interval was characterized by a very low level of geomagnetic activity $($ Dst $=11 \mathrm{nT})$.

The amplitude of a maximum frequency Doppler shift $A_{F}$ at the 'reduced' frequency of $136 \mathrm{MHz}$ was found to be $0.12 \mathrm{~Hz}$. In view of the fact that the shift $F$ is inversely proportional to the sounding frequency squared (Davies, 1969), this corresponds to a Doppler shift at the working frequency of $13.6 \mathrm{MHz}$ and the equivalent oblique-incidence sounding path of about $A_{F}=12 \mathrm{~Hz}$.

The azimuth and elevation $\alpha$ and $\theta$ of the wave vector $\boldsymbol{K}_{\boldsymbol{t}}$ whose horizontal projection is shown in Fig. 1 by a dashed line and is marked by $K_{1}$, are $153^{\circ}$ and $59^{\circ}$, respectively. The horizontal component and the modulus of the phase velocity were found to be $V_{h}=1808 \mathrm{~m} / \mathrm{s}$ and $V_{t}=931 \mathrm{~m} / \mathrm{s}$. The source coordinates at $100 \mathrm{~km}$ altitude were determined as $\phi_{w}=48^{\circ}$ and $\lambda_{w}=66^{\circ}$.

The 'switch-on' delay of the SAW source $\Delta t_{\mathrm{w}}$ with respect to the start time was $264 \mathrm{~s}$. Therefore, it comes as no surprise that the mean velocity of about $V_{a}=1500 \mathrm{~m} / \mathrm{s}$, determined in a usual manner from the response delay with respect to the start, was far greater than the phase velocity $V_{\mathrm{t}}$. Initially, the rocket moved with a supersonic velocity to a reasonably large distance from the launch pad (to $800 \mathrm{~km}$ in the case under consideration), and the SAW source 'switched on' only thereafter.

Similar results for the array (POL2, CHUM, SHAS) and PRN09 were also obtained for failed launch of October 27, 1999. They correspond to the projection of the vector $\boldsymbol{K}_{2}$ in Fig. 1, and to the Table 1. One can only note that the SAW amplitude was by a factor of 4-5 smaller than that for launch of July 5, 1999. At an increased level of magnetic activity (Dst $=-80 \mathrm{nT}$ ) this led to a smaller (compared to July 5 , 1999) signal/noise ratio, which, however, did not interfere with obtaining reliable estimates of SAW parameters.

The comparison of the data for standard launch (November 20, 1998) and failed launches showed that SAW parameters were reasonably similar, irrespective of the level of geomagnetic disturbance, the season, and the local time.

To convince ourselves that the determination of the main parameters of the SAW form and dynamics is reliable for the launches analyzed here, in the area of the Baikonur Cosmodrome we selected different combinations of three sites out of the sets of GPS stations available to us, and these data were processed with the same processing parameters. Relevant results for Baikonur (including the average results for the sets $\Sigma$ ), presented in Table 1 and in Fig. 1 (SAW source position), show that the values of SAW parameters are similar, which indicates a good stability of the data obtained, irrespective of the GPS array configuration.

\section{Discussion}

In spite of the difference of the season, and the level of geomagnetic disturbance, the ionospheric response for all launches has a period $T$ is $270-360$ s, and the amplitude exceeds the standard deviation of background TEC fluctuations in this range of periods under quiet and moderate geomagnetic conditions, by a factor of $2-5$ as a minimum.

Our measurements of the period and amplitude of the SAW response are in good agreement with frequency Doppler shifts measured in the HF range during SPACE SHUTtLE 
launches on February 28, 1990 and April 28, 1991 (Jacobson and Carlos, 1994), as well as with corresponding estimates of a maximum shift $F$ reported by Nagorsky (1999) for oblique HF radio path during rocket launches from Baikonur Cosmodrome. They are also similar to the estimates obtained by Li et al. (1994) using the transionospheric VHF radio signal from geostationary satellite MARECS-B2 during SpaCE ShutTle launches on October 18, 1993 (STS-58) and February 3, 1994 (STS-60).

As pointed out in the Introduction, some researchers report markedly different values of the SAW propagation velocityup to several thousand $\mathrm{m} / \mathrm{s}$, which exceeds the sound velocity at SAW propagation heights in the atmosphere. The horizontal component of the phase velocity $V_{h}$ was in essence determined in such studies. At different values of elevation of the wave vector $\boldsymbol{K}_{\boldsymbol{t}}$, the velocity $V_{h}$ corresponds to markedly different values of the modules of the phase velocity $V_{t}$.

The use of the method proposed in this paper makes the determination of the angular characteristics of the wave vector $\boldsymbol{K}_{t}$ possible and, accordingly, the estimation of $V_{t}$. According to our data (Table 1), the elevation of the SAW wave vector varied from $45^{\circ}-60^{\circ}$, and the SAW phase velocity $V_{\mathrm{t}}$ was in the range of from 900 to $1200 \mathrm{~m} / \mathrm{s}$. We determine the phase velocity of the line of equal TEC at the height of the ionospheric $F$ region maximum which makes the main contribution to variations in TEC between the receiver and the GPS satellite and corresponds to the region of maximum sensitivity of the method. Since $V_{\mathrm{t}}$ approaches the sound velocity at these altitudes (Li et al., 1994), this makes it possible to identify the sound nature of a TEC perturbation.

The position of the SAW source, calculated by neglecting refraction corrections, corresponds to the segment of the PROTON trajectory (Fig. 1) within distances of at least 700 $900 \mathrm{~km}$ from the launch pad. This is consistent with the 'switch-on' delay $\Delta t_{\mathrm{w}}$ of the source which is $250-300 \mathrm{~s}$ for the Proton. As it is evident from our data, the calculated position of the SAW source for rocket launches does not coincide with the position of the launch pad. At the same time the source location is in reasonably good agreement with that of horizontal projections of rocket trajectories (Fig. 1).

Our data are in better agreement with the mechanism substantiated by Li et al. (1994), Calais and Minster (1996), and Nagorsky (1999). They believe that the generation of SAW occurs during a nearly horizontal travel of the rocket with the operating engine along the acceleration segment of the trajectory, at the lower atmospheric heights of 100-130 km. The rocket travels this segment with supersonic velocity at $100-300 \mathrm{~s}$ of its flight at a distance of at least $500 \mathrm{~km}$ from the launch pad. As soon as it ascends to an altitude of about $100 \mathrm{~km}$, the SAW source is 'switched on'.

Acknowledgments. We are grateful to E. A. Ponomarev, V. V. Yevstafiev, A. M. Uralov, P. M. Nagorsky, N. N. Klimov, and A. D. Kalikhman for their interest in this study, helpful advice and active participation in discussions. Thanks are also due to V. G. Mikhalkosky for his assistance in preparing the English version of the manuscript. Finally, the authors wish to thank the referees for valuable suggestions which greatly improved the presentation of this paper. This work was done with support under RFBR grant of leading scientific schools of the Russian Federation No. 00-1598509 and Russian Foundation for Basic Research (grant 99-0564753), as well as under grant 1999 of the RF Ministry of Education (Minvuz), under direction of B. O. Vugmeister.

\section{References}

Afraimovich, E. L., A. I. Terechov, M. Yu. Udodov, and S. V. Fridman, Refraction distortions of transionospheric radio signals caused by changes in a regular ionosphere and by travelling ionospheric disturbances, $J$. Atmos. and Solar-Terr. Phys., 54, 1013-1020, 1992.

Afraimovich, E. L., K. S. Palamartchouk, and N. P. Perevalova, GPS radio interferometry of travelling ionospheric disturbances, J. Atmos. and Solar-Terr. Phys., 60, 1205-1223, 1998.

Afraimovich, E. L., O. N. Boitman, E. I. Zhovty, A. D. Kalikhman, and T. G. Pirog, Dynamics and anisotropy of traveling ionospheric disturbances as deduced from transionospheric sounding data, Radio Sci., 34, 477-487, 1999.

Arendt, P. R., Ionospheric undulations following "Appolo-14" launching, Nature, 231, 438-439, 1971.

Bertel, L., F. Bertin, and J. Testud, De la mesure du contenu électronique intégré appliquée à l'observation des ondes de gravité de moyenne échelle, J. Atmos. and Solar-Terr. Phys., 38, 261-270, 1976.

Calais, E. and J. B. Minster, GPS detection of ionospheric perturbations following the January 1994, Northridge earthquake, Geophys. Res. Lett., 22, 1045-1048, 1995.

Calais, E. and J. B. Minster, GPS detection of ionospheric perturbations following a Space Shuttle ascent, Geophys. Res. Lett., 23, 1897-1900, 1996.

Calais, E., B. J. Minster, M. A. Hofton, and M. A. H. Hedlin, Ionospheric signature of surface mine blasts from Global Positioning System measurements, Geophys. J. Int., 132, 191-202, 1998.

Davies, K., Ionospheric Radio Waves, Blaisdell Publishing Company, A Division of Ginn and Company, Waltham, Massachusetts-Totonto-London, 1969.

Fitzgerald, T. J., Observations of total electron content perturbations on GPS signals caused by a ground level explosion, J. Atmos. and SolarTerr. Phys., 59, 829-834, 1997.

Hofmann-Wellenhof, B., H. Lichtenegger, and J. Collins, Global Positioning System: Theory and Practice, 327 pp., Springer-Verlag, Wien, New York 1992.

Jacobson, A. R. and R. C. Carlos, Observations of acoustic-gravity waves in the thermosphere following Space Shuttle ascents, J. Atmos. and SolarTerr. Phys., 56, 525-528, 1994.

Karlov, V. D., S. I. Kozlov, and G. N. Tkachev, Large-scale disturbances in the ionosphere produced by rocket flight with the operating engine, Kosmicheskiye issledovaniya, 18, 266-277, 1980.

Klobuchar, J. A., Real-time ionospheric science: The new reality, Radio Sci., 32, 1943-1952, 1997.

Li, Y. Q., A. R. Jacobson, R. C. Carlos, R. S. Massey, Y. N. Taranenko, and $\mathrm{G}$. Wu, The blast wave of the Shuttle plume at ionospheric heights, Geophys. Res. Lett., 21, 2737-2740, 1994.

Mendillo, M., The effects of rocket launches of the ionosphere, Adv. Space Res., 1, 275-290, 1981 .

Mendillo, M., Modification of the ionosphere by large space vehicles, $A d v$. Space Res., 2, 150-159, 1982.

Mercier, C. and A. R. Jacobson, Observations of atmospheric gravity waves by radio interferometry: are results biased by the observational technique?, Ann. Géophys., 15, 430-442, 1997.

Nagorsky, P. M., Analysis of the HF radio signal response to ionospehric plasma disturbances caused by shock acoustic waves, Izv. VUZov Radiofizika, 42, 36-44, 1999.

Noble, S. T., A large-amplitude traveling ionospheric disturbance exited by the Space Shuttle during launch, J. Geophys. Res., 95, 19,037-19,044, 1990 .

E. L. Afraimovich (e-mail: afra@iszf.irk.ru), E. A. Kosogorov, K. S Palamarchouk, N. P. Perevalova, and A. V. Plotnikov 